\title{
Development of a disease severity scoring system for patients with
} Pompe disease

\author{
E Giannini*1, K Berger ${ }^{2}$, A van der Ploeg ${ }^{3}$, L Case ${ }^{4}$, C Dandrea ${ }^{5}$, P Kishnani ${ }^{4}$ \\ and D Marsden ${ }^{5}$
}

\begin{abstract}
Address: ${ }^{1}$ Cincinnati Children's Hospital, Cincinnati, OH, USA, ${ }^{2}$ NYU School of Medicine, New York, NY, USA, ${ }^{3}$ Sophia Children's Hospital, Rotterdam, Netherlands, ${ }^{4}$ Duke University Medical Center, Durham, NC, USA and ${ }^{5}$ Genzyme Corporation, Cambridge, MA, USA

* Corresponding author
\end{abstract}

\author{
from 15th Paediatric Rheumatology European Society (PreS) Congress \\ London, UK. 14-17 September 2008 \\ Published: 15 September 2008 \\ Pediatric Rheumatology 2008, 6(SuppI I):PI58 doi:10.1 186/1546-0096-6-SI-PI58
}

This abstract is available from: http://www.ped-rheum.com/content/6/SI/PI58

(c) 2008 Giannini et al; licensee BioMed Central Ltd.

\section{Introduction}

A Disease Severity Scoring System (DS3) measures disease burden in patients. It consists of critical health domains, each described by relevant clinical assessment(s) quantified via reliable, valid and feasible methods. DS3s are particularly useful in rare, heterogeneous diseases in which evaluating severity and prognosis is difficult. Properly configured, a DS3 provides inter- and intra-patient comparisons through time across critical organ systems. A DS3 is being developed for Pompe disease, a rare, autosomal recessive, and heterogenous, neuromuscular disorder.

\section{Description}

Experts were assembled to identify critical Pompe disease health domains. A broader "Delphi" physician group helped capture standard medical practice(s) for severity measurement within each critical domain: Cardiac, Respiratory, Proximal Muscle, Physician Reported Outcomes and Patient Reported

\section{Outcomes}

Within each domain, 1-2 clinical assessments were identified. To test this preliminary model, 9 cases from the Pompe Registry representing a severity spectrum were scored.

\section{Results}

Results were compared to results from a blinded small expert group assessment of the cases using a scale similar to the Clinical Global Impression (CGI) Severity scale, yielding a 0.93 coefficient of correlation, indicating preliminary DS3 consistency with expert opinion, suggesting preliminary DS3 validity, reliability and relevance. Validity and reliability testing are being completed with standardized methods.

\section{Conclusion}

Preliminary results indicate the Pompe DS3 model will help standardize disease terminology and highlight key clinical assessments to quantify disease severity. This tool can become a universal disease "staging" system that permits more exact prediction of important disease outcomes and identify the need for specific medical interventions. 